\title{
Study on Differential Regenerative Braking Torque Control to Increase the Stability of the Small Electric Vehicle with Four In-Wheel Motors
}

\author{
N. M. $\mathrm{Ali}^{1}$, M. H. Peeie ${ }^{1, *}, M . A . H . \mathrm{Rasid}^{1}, H . \mathrm{Ogino}^{2}$ \\ ${ }^{1}$ Faculty of Mechanical Engineering, Universiti Malaysia Pahang, 26600 Pekan, Pahang, Malaysia \\ ${ }^{2}$ Faculty of Prime Mover Engineering, Tokai University, 4-1-1 Kitakaname, Hiratsuka-Shi, 259-1292 \\ Hiratsuka Prefecture, Japan
}

\begin{abstract}
Based on the advantages of the electric motor such as fast and precise torque response, the performance of the electric vehicle (EV) can be improved. During braking or driving on the cornering, the vehicle will over steer or under steer if a car turns by more or less than the amount commanded by the driver. To improve the stability of the small EV with four in-wheel motors, the differential regenerative braking torque control is proposed. In this system, the regenerative braking torque at each wheel will be controlled individually based on the value of slip ratio. If the slip ratio is greater than the optimum value, the regenerative brake will turn off. In this situation, the electric motor will not produce the regenerative braking torque. Conversely, if the slip ratio lower than the optimum value, the regenerative brake will turn on and the electric motor will generate the regenerative braking torque. In the numerical analysis, to investigate the effectiveness of the proposed model, the road condition is set to an icy road on the left tire and dry asphalt on the right tire. From the simulation results, the differential regenerative braking torque control can prevent the tire from lock-up and avoid the vehicle from skidding.
\end{abstract}

\section{Introduction}

In recent years, due to the environmental problems and growing needs of energy, electric vehicles (EVs) are gradually gaining attention to the consumers and manufacturers [1]. The driving system of the EV is the electric motor and there are several advantages of the electric motor such as fast torque response, the generated torque can be detected accurately and motor can be detached on each wheel [2]. Based on these advantages, more effective anti-skid brake system and traction control system (TCS) can be developed on EV.

Over the past years, many studies on anti-lock braking system (ABS) and TCS focuses on to prevent the tire from lock up and vehicle from skidding by controlling the value of slip ratio [3-7]. The optimum slip ratio is important to ensure that the tire can develop a

\footnotetext{
* Corresponding author: mheerwan@ump.edu.my
} 
sufficient high braking force to stop the vehicle [8]. Cai et al have designed two anti-skid controllers; a rule-based controller and a fuzzy logic controller to control the motor torque [9]. In that study, the slip level can be estimated by calculating the ratio of wheel acceleration to motor torque. S. Sakai et al have compared the electric motor with hydraulic brake system as an actuator of ABS [10]. They used a model following control (MFC) as a control algorithm in their comparison.

The research of skid control on small EV have been developed by S. Hasegawa et al, H. Ogino et al and M. H. Peeie et al [11], [12], [13]. S.Hasegawa have examined the braking system of the small EV with two in-wheel motors. From their study, due to the space limitation on the driven tire (rear tire), the hydraulic unit of ABS cannot be installed. ABS only on the front tire and during braking on the slippery road, the driven tire is locked up [11]. To improve the safety of the small EV with the hydraulic-mechanical hybrid brake system, $\mathrm{H}$. Ogino et al have developed a regenerative braking system for a small EV with two in-wheel motors [12]. The ABS system was installed on the front tire and the regenerative brake on the rear tire. Their study shows that the regenerative brake can be controlled to prevent the tire from lock up during braking on the slippery road. M. H. Peeie et al. have developed the regenerative brake timing control to increase safety and stability of the small EV [13]. During braking on critical conditions (icy road and cornering), regenerative brake timing control can prevent the tire from lock up and vehicle from skidding. That model also can improve the steer performance of the vehicle.

In this study, the differential regenerative braking torque control is proposed to improve the stability of the small EV with four in-wheel motors. The control method of the differential regenerative braking torque control is based on the slip ratio on each tire. To examine the effectiveness of the proposed model, the road condition is set to an icy road on the left side and dry asphalt on the right side. The simulation result shows that the differential regenerative braking torque control can improve the stability of the small EV with four in-wheel motors.

\section{Numerical analysis}

\subsection{Vehicle model}

The analysis vehicle model in this study is Toyota COMS, model AK10E-PC. The driving system of this vehicle is two in-wheel motors at the both rear tires. In the numerical analysis, to design the small EV with four in-wheel motors, another two in-wheel motors have been added to the front tires. Figure 1 shows the vehicle model and the specifications of the vehicle as in Table 1 .

\subsection{Slip phenomenon}

In this study, the control method of the differential regenerative braking torque control is based on the relation between slip ratio and friction coefficient. The equation of slip ratio during braking as below:

$$
\rho=\frac{u-r \omega}{r \omega}
$$

where $u$ is the vehicle velocity, $r$ is the radius of the tire and $\omega$ is the angular velocity of the tire. 
The relations between slip ratio and friction coefficient on the dry asphalt and icy road are illustrated in Figure. 2 [13]. Skid phenomenon will occur when the large driving torque suddenly drops to 0 . In this situation, the braking force and the side force generation will disappear and the slip ratio will rapidly increase towards 1.0 . The vehicle will become unstable and skidding.

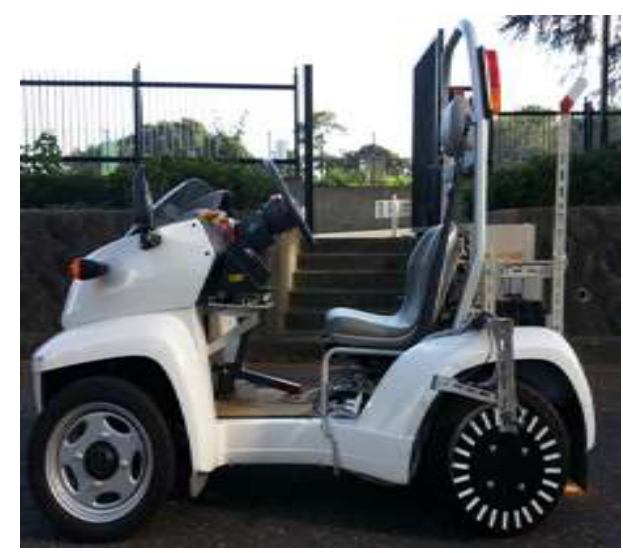

Fig. 1. Analysis vehicle model.

Table 1. Specifications of the analysis vehicle model.

\begin{tabular}{|l|l|}
\hline Vehicle mass & $361.9(\mathrm{~kg})$ \\
\hline Maximum speed & $60(\mathrm{~km} / \mathrm{h})$ \\
\hline Height of centre of gravity & $0.105(\mathrm{~m})$ \\
\hline Tread front & $0.840(\mathrm{~m})$ \\
\hline Tread rear & $0.815(\mathrm{~m})$ \\
\hline Wheelbase & $1.280(\mathrm{~m})$ \\
\hline Inertia of front and rear tire & $2.530\left(\mathrm{kgm}^{2}\right)$ \\
\hline Driving system & 4 in-wheel motors \\
\hline Lead acid batteries & 6 set, $12(\mathrm{~V})$ \\
\hline
\end{tabular}

From Figure. 2, the optimum value of the slip ratio for an icy road is between 0.2 to 0.3 . In this range, the tire can develop a sufficient high braking force to stop the vehicle and prevent the vehicle from skidding.

\subsection{Regenerative braking torque control}

Figure 3 shows the ideal braking force distribution used to determine the percentage of regenerative brake force and mechanical brake force [8]. From the actual brake curve, the maximum degree of rear brake force can be measured by taking the front brake force produced when the coefficient of friction is in maximum value. The equation of ideal braking force for the front and rear tyres are as below:

$$
F_{\text {mecha }}+F_{\text {reg }}=F_{\text {ideal }}
$$

where, $F_{\text {mecha }}$ is the mechanical braking force, $F_{\text {reg }}$ is the regenerative braking force and $F_{\text {ideal }}$ is an ideal braking force. 
During braking on an icy road, the regenerative braking torque control is proposed to improve the braking performance of the small EV. In this model, the regenerative braking torque control will function similarly as ABS, which is based on the slip ratio, $\rho$ of the tire. From Figure 2, if $\rho$ larger than 0.3 , the in-wheel motor turns off and the vehicle in the neutral condition. In this situation, the in-wheel motors do not produce regenerative braking torque. The braking force on the tire is only mechanical braking force. However, if $\rho$ becomes lower than 0.2 , the in-wheel motor turns on and produce the regenerative braking torque to regain the ideal braking force.

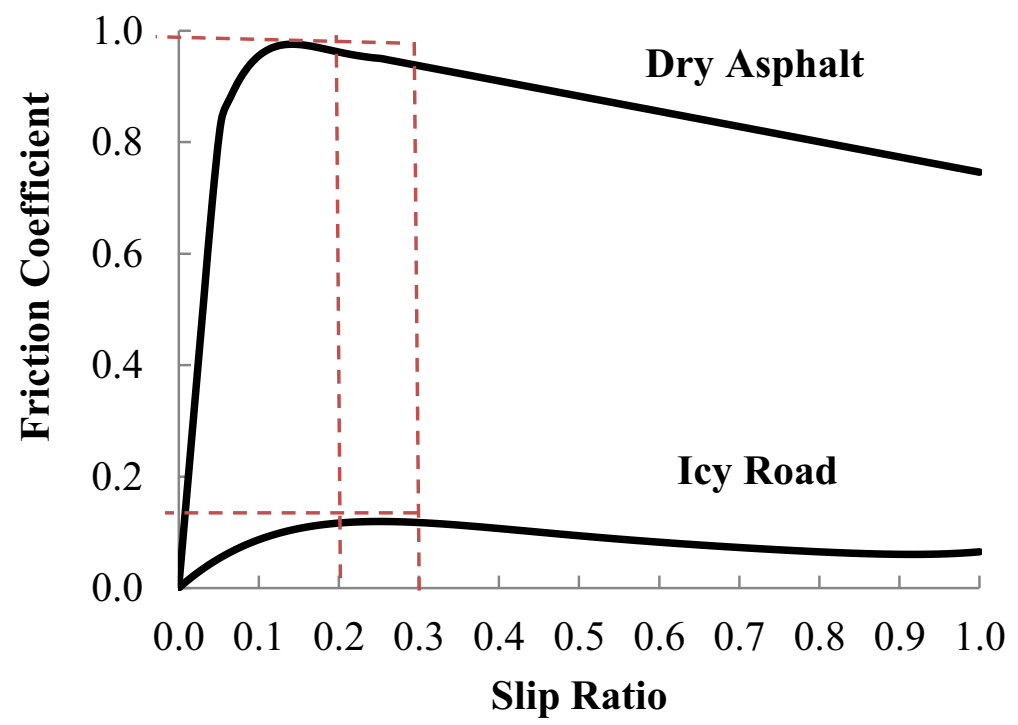

Fig. 2. Friction coefficient and slip ratio on the dry asphalt and icy road.

\subsection{Dynamics equation of motion}

Figure 4 shows the force vector at each wheel for the construction of equations of motion. The equation of motion in longitudinal and lateral direction and the equation of yaw motion are shown as below:

$$
\begin{gathered}
m\left(\frac{d u}{d t}-v \gamma\right)=\left(-X_{f r}-X_{f l}\right) \cos \theta+\left(-Y_{f r}-Y_{f l}\right) \sin \theta-X_{r r}-X_{r l} \\
m\left(\frac{d v}{d t}+u \Upsilon\right)=\left(Y_{f r}+Y_{f l}\right) \cos \theta+\left(-X_{f r}-X_{f l}\right) \sin \theta+Y_{r r}+Y_{r l}
\end{gathered}
$$

$I \frac{d \gamma}{d t}=$

$l_{f}\left(Y_{f r} \cos \theta+Y_{f l} \cos \theta-X_{f r} \sin \theta-X_{f l} \sin \theta\right)-l_{r}\left(Y_{r r}+Y_{r l}\right)+\frac{d_{f}}{2}\left(-X_{f r} \cos \theta+\right.$

$\left.X_{f l} \cos \theta-\quad Y_{f r} \sin \theta+Y_{f l} \sin \theta\right)+\frac{d_{r}}{2}\left(-X_{r r}+X_{r l}\right)$ 
where $u$ and $v$ is the velocity of the vehicle in longitudinal and lateral axis, $\Upsilon$ is the vehicle yaw rotational speed, $X_{f r}, X_{f l}, X_{r r}, X_{r l}$ are the friction force while $Y_{f r}, Y_{f l}, Y_{r r}, Y_{r l}$ are the cornering force.

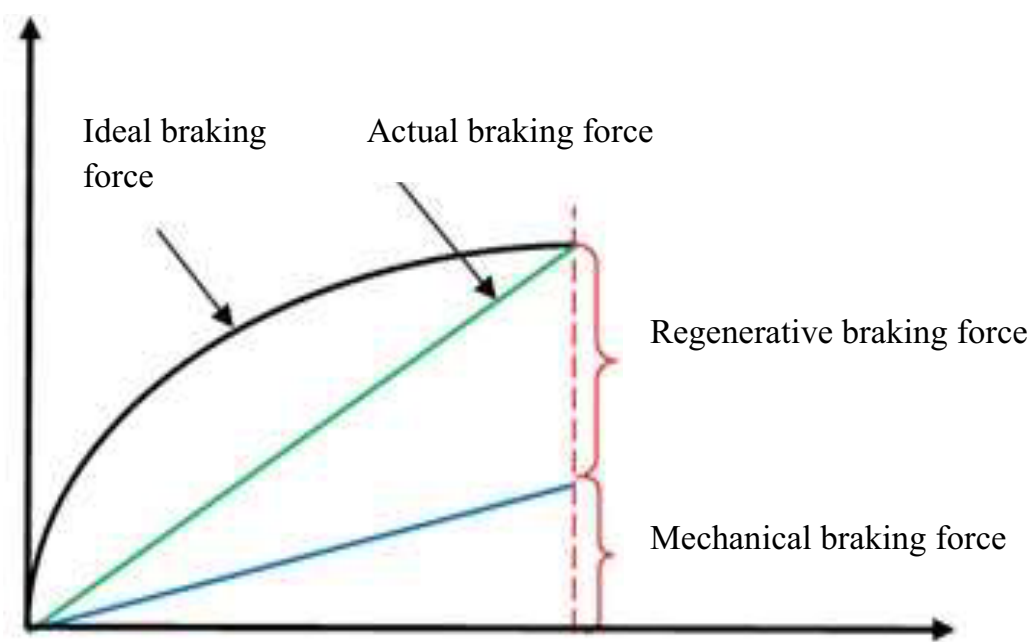

Fig. 3. Ideal braking force distribution.

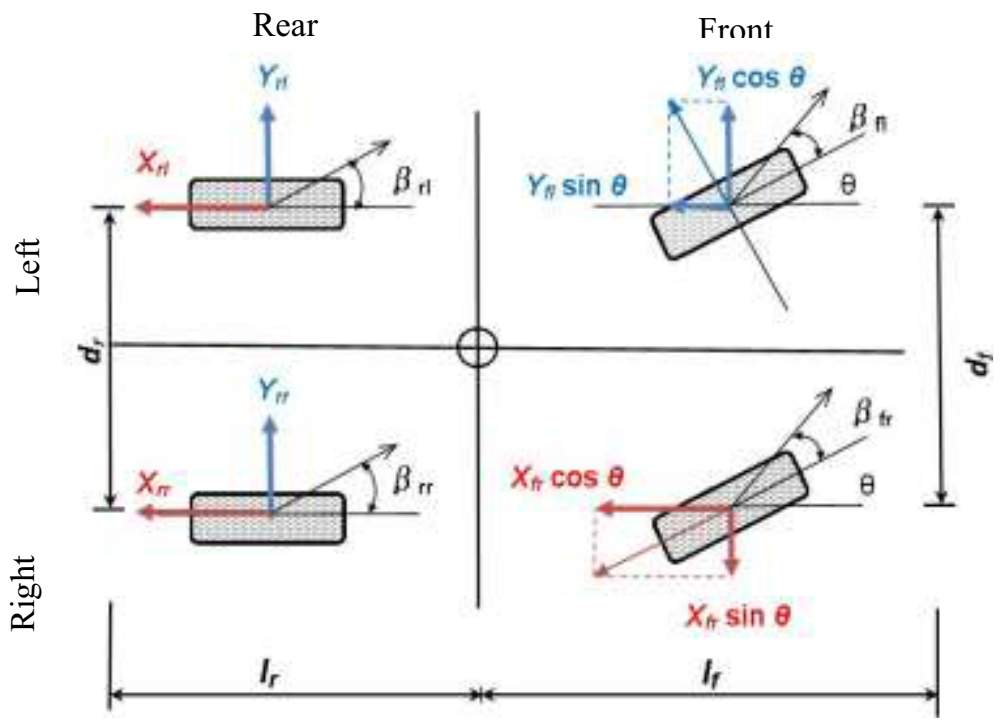

Fig. 4. Force diagram of the vehicle.

The brush tire model has been used in the simulation to measure the friction force, $X_{f r}$, $X_{f l}, X_{r r}, X_{r l}$ and cornering force, $Y_{f r}, Y_{f l}, Y_{r r}, Y_{r l}$. This model allows elastic deformation in both the longitudinal and lateral directions. The equation of friction force and cornering force during braking as below: 


$$
\begin{aligned}
& \xi_{p}=1-\frac{K_{s} \lambda}{3 \mu W_{z}(1-\rho)} \\
& \lambda=\sqrt{\rho^{2}+\left(\frac{K_{\beta}}{K_{s}}\right)^{2} \tan ^{2} \beta_{T}} \\
& K_{s}=\frac{b l_{T}{ }^{2}}{2} K_{x} \quad, \quad K_{\beta}=\frac{b l_{T}{ }^{2}}{2} K_{y} \\
& X f r, X f l, X r r, X r l \begin{cases}=-\frac{K_{s} \rho}{1-\rho} \xi_{p}{ }^{2}-6 \mu W_{z} \frac{\rho}{\lambda}\left(\frac{1}{6}-\frac{1}{2} \xi_{p}{ }^{2}+\frac{1}{3} \xi_{p}{ }^{3}\right) & \left(\xi_{p}>0\right) \\
=-\mu W_{z} \frac{\rho}{\lambda} & \left(\xi_{p}<0\right)\end{cases} \\
& Y f r, Y f l, Y r r, Y r l \begin{cases}=-\frac{K_{\beta} \tan \beta_{T}}{1-\rho} \xi_{p}{ }^{2}-6 \mu W_{z} \frac{K_{\beta} \tan \beta_{T}}{K_{s} \lambda}\left(\frac{1}{6}-\frac{1}{2} \xi_{p}{ }^{2}+\frac{1}{3} \xi_{p}{ }^{3}\right) & \left(\xi_{p}>0\right) \\
=-\mu W_{z} \frac{K_{\beta} \tan \beta_{T}}{K_{S} \lambda} & \left(\xi_{p}<0\right)\end{cases}
\end{aligned}
$$

Where, $b, l$ is the width and length of the interacted tyre surface, $K_{x}, K_{y}$ are the rigidness of tyre in longitudinal and lateral axis, and $\beta_{T}$ is the side slip angle of the tyre. Based on the analysis vehicle model, the value of $b, l, K_{x}$ and $K_{y}$ are $10 \mathrm{~cm}, 15 \mathrm{~cm}$, $3.3 \times 10^{7} \mathrm{~N} / \mathrm{m}^{3}$ and $3.3 \times 10^{7} \mathrm{~N} / \mathrm{m}^{3}$ respectively.

\subsection{Calculation procedures}

When a braking torque $T_{B}$ is applied to the tyre, a corresponding torque friction of the tyre $T_{f}$ is developed on the tyre ground contact patch, which acts in the opposite direction of the applied braking torque $T_{B}$. The difference between $T_{B}$ and $T_{F}$ causes an angular acceleration $\dot{\omega}$ of the tyre:

$$
\left\{\begin{array}{l}
T_{f f r}-T_{B f r}=I_{f r} \frac{d \omega_{f r}}{d t} \\
T_{f r r}-T_{B r r}=I_{r r} \frac{d \omega_{r r}}{d t}
\end{array}, \quad\left\{\begin{array}{l}
T_{f f l}-T_{B f l}=I_{f l} \frac{d \omega_{f l}}{d t} \\
T_{f r r}-T_{B r r}=I_{r r} \frac{d \omega_{r r}}{d t}
\end{array}\right.\right.
$$

where $I$ is the inertia of the tyre. The inertia moment of the front tyre is $0.43 \mathrm{kgm}^{2}$ and the inertia moment of the rear tyre is $2.53 \mathrm{kgm}^{2}$ [1].

During braking, the in-wheel motor will produce the regenerative braking torque. Then, the total braking torque for on each tyre is the sum of the mechanical braking torque and the regenerative braking torque. Equation (9) shows the braking torque at each tyre.

$$
\left\{\begin{array} { l } 
{ T _ { B f r } = B E F _ { f r } \times r _ { f r } B _ { f r } } \\
{ T _ { B f l } = B E F _ { f l } \times r _ { f l } B _ { f l } }
\end{array} \quad \left\{\begin{array}{l}
T_{B r r}=B E F_{r r} \times r_{r r} B_{r r}+T_{R r} \\
T_{B r l}=B E F_{r l} \times r_{r l} B_{r l}+T_{R l}
\end{array}\right.\right.
$$

Where $B E F$ is braking efficiency factor and the value of $B E F$ is 1.5 .

If $\rho$ is not in the optimum range ( 0.2 to 0.3$)$, the ABS control unit will start to operate to 
control the braking pressure in the master cylinder. The braking pressure from the master cylinder will be directed to the front wheel cylinder and rear power cylinder. Without a time delay response from the master cylinder to the front wheel cylinder, and ABS is enough to maximise the slip ratio and cornering force of the front tyre. However, for the rear braking system, due to the rigidness of the mechanical braking system, a time delay response occurred during ABS operational. To compensate for the loss of friction force, the in-wheel motor will produce the regenerative braking torque. The regenerative braking control timing will be operational when the value of $\rho$ is not in the optimum range. From the operation of the ABS and regenerative brake control timing, we can keep the optimum value of $\rho$ and we also can maximise the braking force and cornering force, thus assuring the stability of the vehicle.

\section{Results and discussion}

\subsection{Effect of the regenerative braking without electric motor control}

In the first part of this study, the effect of the regenerative braking without electric motor control have been examined. The right side of the road is dry asphalt and the left side is an icy road. Figure 5 shows the tire rotational speed (uFL, uFR, uRL, uRR) and vehicle speed (u) when differential regenerative braking torque control does not apply to all tires. From this result, the rotational speed of the right tires (uFR, uRR) almost similar with the speed of the vehicle. However, due to the low friction between road and tire on the left tires (uFL, $\mathrm{uRL}$ ), the rotational speed of uFL and uRL was decreased rapidly from $8.3 \mathrm{~m} / \mathrm{s}$ to $1 \mathrm{~m} / \mathrm{s}$ in 4 seconds. After 4 seconds, the rotational speed of uFL and uRL was similar with the speed of $\mathrm{u}, \mathrm{uFR}$ and $\mathrm{uRR}$. It means that in the low-speed condition, the regenerative braking force produced by the electric motor is every small and not affect the slip of the tire.

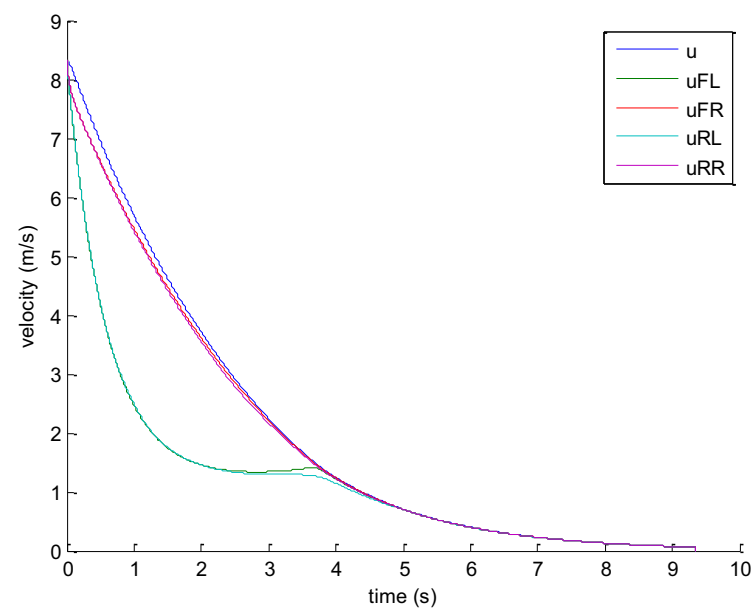

Fig. 5. Deceleration of the vehicle and tire without differential regenerative braking torque control. 
The skidding condition of the vehicle can be measured from the slip ratio of the tire in Figure 6. From Figure 6, the slip ratio of the right tires (slip ratio FR, slip ratio RR), are very small. When braking on the dry asphalt, due to the high friction between the tire and road, the optimum braking force can be applied to stop the tire from rotating. However, on the icy road condition, the slip ratio of the tire (slip ratio FL, slip ratio RL) are increases rapidly from 0 to 0.6 in 1.5 seconds. Start from 1.5 seconds to 4 seconds, the slip ratio FL and RL were decreased rapidly to 0.5 . The regenerative braking torque produced by the electric motor is proportional to the speed of the tire. Without regenerative braking torque control, in a high-speed situation, the tire will slip. On the other hand, in a low-speed condition, which is start from 4 seconds, the slip ratio is very small.

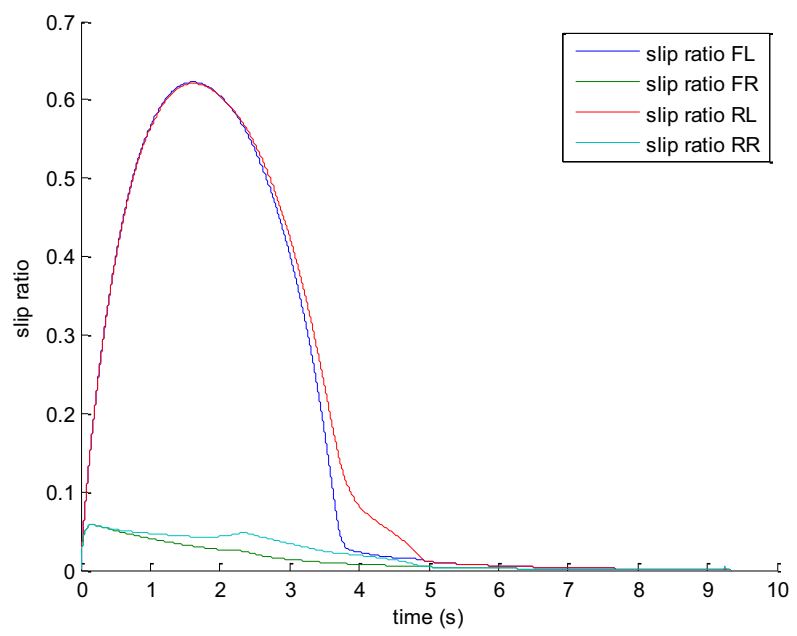

Fig. 6. Slip ratio of the tire without differential regenerative braking torque control.

\subsection{Effect of the differential regenerative braking torque control}

The second part of this study is to investigate the effect of the differential regenerative braking torque control on the dry asphalt and icy road. Figure 7 shows the deceleration speed of the vehicle $(u)$ and tire (uFL, uFR, uRL, uRR). From this result, the rotational speed of the right tires (uFR, uRR) was decreased approximately to the speed of the vehicle. On the dry asphalt condition, the friction between contact patch of the tire and road are high, and the regenerative braking force produced by the electric motor can stop the tire without any slip. However, for the left tires (uFL, uRL), the deceleration of the tires are fluctuated start from the beginning until 3.5 seconds. In a high-speed situation, when braking on an icy road with differential regenerative braking torque control, the electric motor will turn off and turn on based on the slip ratio of the tire. From this mechanism, the rotational speed of the tire will fluctuate. From 3.5 seconds, the rotational speed of the uFL and $\mathrm{uRL}$ are similar with $\mathrm{u}, \mathrm{uFR}$ and $\mathrm{uRR}$. This situation occurs because, in a low-speed condition, the regenerative braking force is minimised and no slip on the tire. 


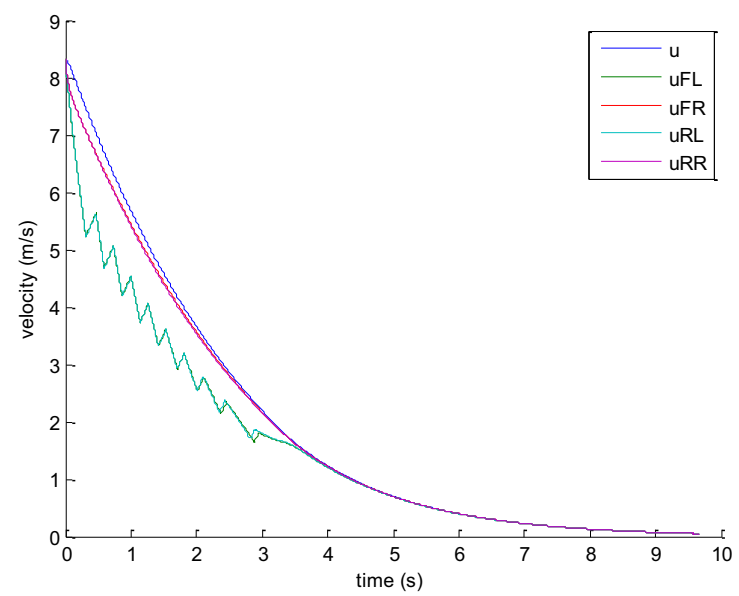

Fig. 7. Deceleration of the vehicle and tire when differential regenerative braking torque control is applied.

The slip ratio of the tire (slip ratio FL, FR, RL,RR) are shown in Figure 8. From this result, the slip ratio FR and RR are very small. On a dry asphalt condition, there is no slip on the tire. However, on an icy road, the slip ratio FL and RL have fluctuated in the 3.2 seconds. In this condition, the controller sends the signal to the electric motor to control the regenerative braking torque on each wheel to prevent the tire from lock up. After 3.2 seconds, there is no signal from the controller to control the regenerative braking torque because the rotational speed of the tire is very small.

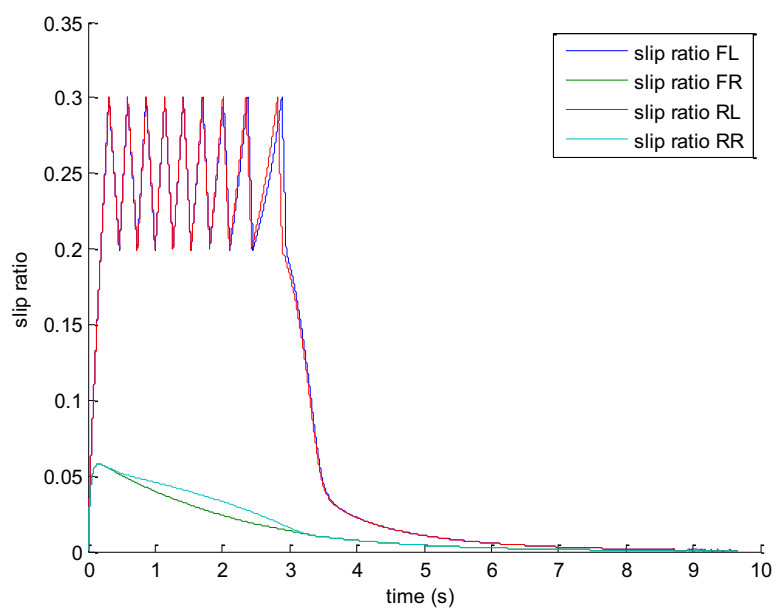

Fig. 8. Slip ratio when differential regenerative braking torque control is applied.

\section{Conclusions}

In this paper, the differential regenerative braking torque controller is developed to increase the stability of four small EV with four in-wheel motors. To investigate the effectiveness of 
the differential regenerative braking torque control, the simulation model has been developed in the Matlab-Simulink. In the simulation, the right side of the road is set to the dry asphalt and the left side of the road is set to an icy road. From the simulation result, when braking on the different road condition for the right and left side, the differential regenerative braking torque control can avoid the tire from lock up. Besides, it also can prevent the vehicle from skidding and increase the stability of the vehicle.

The authors would like to thank Universiti Malaysia Pahang (UMP) for providing laboratory facilities and financial assistance under project no. RDU160312. Special thanks to Ministry of Higher Education Malaysia for providing Research Acculturation Grant Scheme (RAGS) under project no RDU151403.

\section{References}

1. Y. O. Mohamad Heerwan Bin Peeie, Hirohiko Ogino, Skid Control of Small Electric Vehicle, Proc. 2013 IEEE Conf. Syst. Process Control, Dec, pp. 13-15. (2013).

2. H. F. Toru Suzuki, Slip Ratio Estimation and Regenerative Brake Control without Detection of Vehicle Velocity at Urgent Brake Turning, $11^{\text {th }}$ IEEE International Workshop on Advanced Motion Control, (2010).

3. D. Peng, Y. Zhang, C. Yin, and J. Zhang, Combined Control of a Regenerative Braking and Anti-lock Braking System for Hybrid Electric Vehicles, vol. 9, no. 6, pp. 749-757. (2008).

4. S. Sakai and Y. Hori, Road Condition Estimation for Traction Control in Electric Vehicle, IEEE International Symposium on Industrial Electronics, pp. 973-978. (1999).

5. M. Wu and M. Shih, Simulated and experimental study of hydraulic anti-lock braking system using sliding-mode PWM control, Mechatronics, vol. 13, no. 4, pp. 331-351. (2003).

6. H. Fujimoto, T. Saito, a. Tsumasaka, and T. Noguchi, Motion control and road condition estimation of electric vehicles with two in-wheel motors, Proc. 2004 IEEE Int. Conf. Control Appl. 2004., vol. 2, pp. 1266-1271. (2004).

7. J. R. Cho, J. H. Choi, W. S. Yoo, G. J. Kim, and J. S. Woo, Estimation of dry road braking distance considering frictional energy of patterned tires, Finite Elem. Anal. Des., vol. 42, no. 14-15, pp. 1248-1257. (2006).

8. M. H. Peeie, H. Ogino, and Y. Oshinoya, Skid control of small electric vehicle with hydraulic-mechanical hybrid brake system ( Effect of ABS and regenerative brake control on an icy road), Mech. Eng. Journal, Japan Soc. Mech. Eng., vol. 1, no. 5, pp. 1-15. (2014).

9. Z. Cai, C. Ma, and Q. Zhao, Acceleration-to-torque ratio based anti-skid control for electric vehicles, Proc. 2010 IEEE/ASME Int. Conf. Mechatron. Embed. Syst. Appl., pp. 577-581. (2010).

10. S.-I. Sakai, H. Sado, and Y. Hori, Anti-skid control with motor in electric vehicle, 6th Int. Work. Adv. Motion Control Proc., pp. 317-322. (2000).

11. S. Hasegawa and H. Ogino, Research on Skid Control of Small Electric Vehicle with Hydraulic-Mechanical Hybrid Brake System (1 st Report; Hydraulic simulation of antilock brake system for two-wheel models), Proc. Sch. Eng. Tokai Univ., vol. 49, no. 2, pp. 89-94. (2009). 
12. H. Ogino, S. Kobayashi, and S. Hasegawa, Research on Skid Control of Small Electric Vehicle (Effect of Regenerative Braking System in Skidding Condition), Trans. JAPAN Soc. Mech. Eng. Ser. C, vol. 77, no. 784, pp. 4605-4618. (2011).

13. M. H. Bin Peeie, H. Ogino, and Y. Oshinoya, Skid control of a small electric vehicle with two in-wheel motors: simulation model of ABS and regenerative brake control, Int. J. Crashworthiness, vol. 8265, no. April, pp. 1-1., (2016). 